\title{
Evaluación de las ecuaciones de estimación de la tasa de filtrado glomerular basadas en creatinina sérica CKD-EPI y MDRD en pacientes mexicanos adultos. Estudio piloto
}

\author{
José O. Sosa-Ramos ${ }^{1 *}$, Omar A. Jiménez-Garcia², M. del Rosario Cruz-Juanes³, \\ Martha A. Hernández-González ${ }^{4}$ y Rafael Luna-Montalbán ${ }^{1}$
}

${ }^{1}$ Departamento de Medicina Interna; ${ }^{2}$ Departamento de Nefrología, Unidad de Hemodiálisis, Consulta Externa de Nefrología Prediálisis; ${ }^{3}$ Departamento de Medicina Nuclear, Unidad de Medicina Nuclear; ${ }^{4}$ División de Investigación en Salud. UMAE N. ${ }^{0} 1$, Hospital de Especialidades del Centro Médico Nacional del Bajío, IMSS, León, Guanajuato, México

\section{Resumen}

Objetivo: Comparar la funcionalidad de las ecuaciones MDRD (Modification of Diet in Renal Disease Study) y CKD-EPI (Chronic Kidney Disease Epidemiology Collaboration) para la evaluación de la función renal en pacientes adultos mexicanos, usando un renograma dinámico ${ }^{99 m}$ Tc-DTPA (Gates modificado) como estándar de oro. Material y métodos: Estudio observacional, retrospectivo, transversal en 54 pacientes sometidos a renograma con y sin enfermedad renal en el Hospital de Especialidades $N^{\circ} 1$ del Centro Médico Nacional el Bajío del Instituto Mexicano del Seguro Social, León, Guanajuato, México. Se determinaron cuatro categorías de validez: concordancia, sesgo (TFG medida-TFG estimada), precisión (TFG medida-TFG estimada)/TFG medida), exactitud $P$ (30) y $P$ (10) (porcentaje de TFG estimadas alrededor del 30 y el $10 \%$ de la TFG medida). Resultados: Concordancia: $R^{2}=0.6238$ para CKD-EPI contra renograma $(p=0.001)$ y $R^{2}=0.6022$ para MDRD contra renograma $(p=0.029)$. Sesgo medio: CKD-EPI $-11.39 \pm 19.47 \mathrm{ml} / \mathrm{min} / 1.73 \mathrm{~m}^{2}$ (IC 95\%: -16.59 a -6.20 ; $p<0.001$ ), MDRD -7.38 $\pm 19.18 \mathrm{ml} / \mathrm{min} / 1.73 \mathrm{~m}^{2}$ (IC 95\%: -12.49 a -2.26; p 0.008). Precisión media: CKD-EPI -28\% $\pm 57 \%$ (IC 95\%: $-43 \%$ a $-12 \% ; p<0.001$ ), MDRD -19\% \pm 51\% (IC 95\%: -33\% a -6\%; $p=0.008$ ). Exactitud P (30): CKD-EPI 57\%, MDRD 61\% ( $p<0.001)$. Exactitud P (10): CKD-EPI 17\%, MDRD 20\% ( $<$ < 0.001). Conclusiones: Ambas ecuaciones sobreestiman significativamente la TFG. La MDRD mostraría mejor funcionalidad que la CKD-EPI en mexicanos, especialmente con enfermedad renal crónica. Es necesaria la realización de estudios de mayor escala para confirmar nuestros resultados.

Palabras clave: TFG. MDRD. CKD-EPI. Mexicanos. Renograma.

\section{Evaluation of creatinine-based glomerular filtration rate estimation equations CKD-EPI and MDRD in Mexican adult patients, pilot study}

\section{Abstract}

Objective: To compare the performance of modification of diet in renal disease (MDRD) study and chronic kidney disease epidemiology collaboration (CKD-EPI) equations, in renal function evaluation among adult mexican patients, using ${ }^{99 m} T c-D T$ PA Dynamic Renogram (Gates-modified method) as gold-standard. Materials and methods: Observational, retrospective

\section{Correspondencia:}

*José O. Sosa-Ramos,

E-mail: soy_sos@hotmail.com
Fecha de recepción: 11-10-2019

Fecha de aceptación: 06-02-2020

DOI: 10.24875 /NEFRO.20000104
Disponible en internet: 03-04-2020

Nefro Latinoam. 2020;17:43-51 www.nefrologialatinoamericana.com

(3) 2020 Sociedad Latinoamericana de Nefrología e Hipertensión. Publicado por Permanyer. Este es un artículo open access bajo la licencia CC BY-NC-ND (http://creativecommons.org/licenses/by-nc-nd/4.0/). 
Nefro Latinoam. 2020;17

and transversal study with 54 enrolled patients, including CKD and healthy individuals that underwent ${ }^{99 m}$ TC-DTPA Dynamic Renogram in the Hospital de Especialidades \#1 of CMN Bají, IMSS, León, Guanajuato, Mexico. Four categories of validity were chosen: Concordance, Bias (measured glomerular filtration rate [GFR] - estimated GFR), Precision (measured GFR estimated GFR)/measured GFR), Accuracy $P$ (30) and $P$ (10) (percentage of estimated GFR among $30 \%$ and $10 \%$ around measured GFR). Results: Concordance: $R^{2}=0.6238$ for CKD-EPI versus Renogram $(p=0.001)$, and $R^{2}=0.6022$ for MDRD vs Renogram $(p=0.029)$. Mean Bias: CKD-EPI $-11.39 \pm 19.47 \mathrm{~m} / \mathrm{min} / 1.73 \mathrm{~m}^{2}(\mathrm{Cl} 95 \%$ : $-16.59--6.20 ; p<0.001)$, MDRD $-7.38 \pm 19.18 \mathrm{ml} / \mathrm{min} / 1.73 \mathrm{~m}^{2}$ (Cl 95\%: -12.49--2.26; $\left.p=0.008\right)$. Mean Precision: CKD-EPI -28\% \pm 57\% (Cl 95\%: $-43 \%--12 \%$; $p<0.001), M D R D-19 \% \pm 51 \%$ (CI 95\%: $-33 \%--6 \% ; p=0.008)$. Accuracy P (30): CKD-EPI 57\% vs MDRD 61\% ( $p<0.001)$; Accuracy P (10): CKD-EPI 17\% versus MDRD 20\% ( $p<0.001)$. Conclusions: Both equations significantly overestimate GFR. MDRD would show better performance than CKD-EPI in Mexican patients, notably in CKD. Larger scale studies are needed to confirm our results.

Key words: Glomerular filtration rate. Modification of diet in renal disease. Chronic kidney disease epidemiology collaboration. Mexicans. Renogram.

\section{Introducción}

La determinación de la tasa de filtrado glomerular (TFG) es usualmente aceptada como el mejor índice de función renal y representa una de las mejores herramientas para el diagnóstico, clasificación y seguimiento de pacientes identificados con alteraciones en dicha funcionalidad. Así mismo, se emplea frecuentemente en ensayos clínicos para evaluar el desenlace en la función renal, además de poseer por sí sola un valor pronóstico para la mortalidad o el requerimiento de terapia de sustitución renal ${ }^{1-3}$. Sin embargo, su medición es poco práctica por los altos costos, carácter invasivo y complejidad de obtención, por lo que habitualmente solo se emplea con fines de investigación ${ }^{4}$. En consecuencia, se desarrollaron métodos no invasivos de estimación de la tasa de filtrado glomerular, y su uso ha sido ampliamente aceptado debido a su bajo costo, fácil y rápido acceso, así como la buena correlación con la TFG medida por métodos invasivos, que han ido evolucionando de acuerdo con los avances técnicos y resultados de trabajos de investigación de gran escala-12.

Destacan las ecuaciones de estimación de la TFG basadas en la creatinina sérica desarrolladas por los estudios Modification of Diet in Renal Disease Study (Levey, et al. 1999-2005) y Chronic Kidney Disease Epidemiology Collaboration (Levey, et al. 2009), MDRD y CKD-EPI, respectivamente ${ }^{2,13}$; no obstante, tales ecuaciones no se han validado para la población hispana no residente de EE.UU. o Europa. La falta de validación del funcionamiento de tales ecuaciones en otros grupos étnicos o razas, específicamente la hispana, se atribuye al diseño de los estudios que las desarrollaron, especialmente en la selección de la población estudiada, que no necesariamente se correlaciona con el resto de la población mundial. En la MDRD, la mayoría de individuos fueron pacientes de raza blanca, no diabéticos y con tasas de filtrado glomerular menores a $60 \mathrm{ml} / \mathrm{min} / 1.73$ $\mathrm{m}^{2}$, por lo que perdía su funcionalidad en pacientes con mayores tasas y se sobreestimaba la prevalencia de enfermedad renal crónica (ERC). En la CKD-EPI no se incluyeron las «minorías étnicas» como variables, pese a haber sido incluidas en la población analizada, por lo que se anexó este grupo de residentes de EE.UU. a la categoría de «raza blanca», así mismo, se excluyeron los pacientes mayores de 65 años, además, la selección de grupo étnico hispano designado como «latino» fue conglomerado con el nativo americano adjudicando tener el mismo origen antropológico, finalmente, la media del índice de masa muscular fue de de $31 \mathrm{~kg} / \mathrm{m}^{2}(54 \%$ mayor de $\left.30 \mathrm{~kg} / \mathrm{m}^{2}\right)^{14-20}$. Tal sesgo se ha detallado en diversos estudios de validación de dichas ecuaciones sobre distintas poblaciones o grupos étnicos y etarios alrededor del mundo, en algunos de ellos incluso se han propuesto adecuaciones a las ecuaciones para tales regiones ${ }^{21-26}$.

Existen al menos otras dos ecuaciones de estimación de la TFG basadas en la creatinina ampliamente difundidas, la Mayo Clinic MC o cuadrática y la MCRR Rule Reffited, ambas desarrolladas por la Clínica Mayo en 2004, sin embargo, consideran más variables (antropométricas y bioquímicas), lo que las hace un poco menos accesibles que la CKD-EPI y la MDRD (basadas solo en la edad, sexo, raza y niveles de creatinina sérica), y se ha demostrado que no son superiores a estas para el diagnóstico y seguimiento de la ERC ${ }^{27,28}$.

Destaca que escasos estudios se han diseñado con el fin de demostrar el funcionamiento de las ecuaciones de estimación de la TFG basadas en la creatinina sérica en individuos con origen étnico hispano con o sin alteraciones en la función renal. Sobresale uno de ellos realizado en el Instituto Nacional de Ciencias Médicas 
y Nutrición Salvador Zubirán en la Ciudad de México en el año 2014, que identifica discrepancias con los resultados provistos por los estudios CKD-EPI y MDRD en la correlación entre las ecuaciones de estimación de la TFG basadas en la creatinina sérica y la TFG medida en población mexicana sana ${ }^{29}$, sustentando un antecedente, el único, para esta población.

El objetivo del presente estudio fue comparar el funcionamiento de las ecuaciones de estimación de la tasa de filtrado glomerular basadas en la creatinina sérica (CKD-EPI y MDRD) para la evaluación de la función renal y la clasificación e identificación de progresión de la ERC en pacientes mexicanos adultos, utilizando el renograma dinámico ${ }^{99 \mathrm{~m}} \mathrm{Tc}$-DTPA por método de Gates modificado como estándar de oro para la medición de la TFG. Se identificó la presencia de variaciones significativas en sesgo, precisión y exactitud de la estimación de la TFG por medio de tales ecuaciones.

\section{Material y métodos}

Se realizó un estudio observacional, retrospectivo y transversal sobre una muestra de pacientes mexicanos

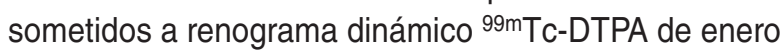
a octubre de 2018 en la Unidad de Medicina Nuclear del Hospital de Especialidades de la Unidad Médica de Alta Especialidad N. ${ }^{\circ} 1$ del Centro Médico Nacional del Bajío del Instituto Mexicano del Seguro Social, León, Guanajuato, México.

Se obtuvieron los datos del registro de estudios realizados de la base de datos de la Unidad de Medicina Nuclear, mediante la búsqueda intencionada de "Gammagrafía Renal ${ }^{99 m}$ Tc-DTPA», así como la base de datos local de estudios de laboratorio para el registro de creatinina sérica.

Se incluyeron todos aquellos pacientes con edad $\geq$ 18 años, sin distinción de sexo, diagnóstico de envío (al ser especificado), unidad de adscripción (envío) ni resultados reportados, y se eliminaron aquellos sujetos que no tuvieran al menos una determinación de niveles de creatinina en sangre de no más de +15 días a partir de la fecha de realización del renograma (sesgo temporal, presente sobre todo en pacientes con lesión renal aguda y uropatía obstructiva).

Se utilizó estadística descriptiva según el nivel de medición de las variables. Los resultados se muestran como frecuencias y porcentajes para las variables categóricas y como medias más desviación estándar (DE) para las variables continuas.

Para el funcionamiento como prueba diagnóstica se determinaron cuatro categorías de validez:
1. Sesgo (diferencia normal): calculado por la diferencia entre la TFG medida y la TFG estimada (TFGm-TFGe).

2.Precisión (diferencia absoluta): porcentaje de sesgo para la comparación entre métodos ([TFGm-TFGe]/ TFGm).

3. Exactitud $P(30)$ y exactitud $P(10)$ : porcentaje de las TFG estimadas que se encuentran en el espacio comprendido en el 30 y $10 \%$, respectivamente, por encima y por debajo de la TFG medida (TFGm $\pm 30 \%$ y $\mathrm{TFGm} \pm 10 \%$ ).

4. Concordancia (acuerdo entre métodos): mediante el estadístico de Bland-Altman y concordancia (Rho) de Pearson.

Se consideraron resultados con signo positivo como infraestimación y con signo negativo como sobreestimación.

Para la clasificación por grados, se agrupó a los sujetos según la TFGe en los distintos grados de acuerdo a la Kidney Disease Improved Global Outcomes (KDIGO 2012) (grado 1 [normal o alto]: > $90 \mathrm{ml} / \mathrm{min} / 1.73 \mathrm{~m}^{2}$; grado 2 [levemente disminuido]: $60-89 \mathrm{ml} / \mathrm{min} / 1.73 \mathrm{~m}^{2}$; grado $3 a$ [leve a moderadamente disminuido]: 45$59 \mathrm{ml} / \mathrm{min} / 1.73 \mathrm{~m}^{2}$; grado $3 \mathrm{~b}$ [moderada a severamente disminuido]: $30-44 \mathrm{ml} / \mathrm{min} / 1.73 \mathrm{~m}^{2}$; grado 4 [severamente disminuido]: $15-29 \mathrm{ml} / \mathrm{min} / 1.73 \mathrm{~m}^{2}$; grado 5 [falla renal]: $<15 \mathrm{ml} / \mathrm{min} / 1.73 \mathrm{~m}^{2}$ ), y se compararon contra la clasificación por TFGm por renograma dinámico ${ }^{99 m} \mathrm{TC}$ DTPA, y se obtuvo también sesgo y precisión. Los valores expresados en esta categoría con signo positivo se consideran como subdiagnóstico y con signo negativo se consideraron como sobrediagnóstico.

Se analizó la concordancia por medio de Rho de Pearson ante distribución normal (prueba de Kolmogorov-Smirnov) e índice de determinación (r2), ANOVA a dos vías entre TFGe por método y TFGm, así como prueba $Z$ a una y dos colas para la comparación entre métodos. Los resultados para la comparación de funcionalidad entre ecuaciones de estimación de TFG fueron analizados con la prueba $T$ pareada para sesgo y sesgo $\%$, con intervalos de confianza al $95 \%$, para la variable exactitud se utilizó la prueba de $\chi 2$. Se consideró significativo un nivel de $p<0.05$. El análisis estadístico se llevó a cabo en el software estadístico IBM SPSS Statics v23.0 y Microsoft Excel 2016.

\section{Resultados}

Se obtuvo el registro de 283 pacientes sometidos a renograma dinámico ${ }^{99 \mathrm{~m} T \mathrm{~T}-\mathrm{DTPA}}$ de enero a octubre de 2018. Fueron excluidos 185 registros por corresponder 
a pacientes pediátricos (menores de 18 años), posteriormente se eliminaron 20 registros por no contar con determinación de creatinina sérica registrada en el sistema electrónico y 24 más por tener estos registros con fechas superiores a +15 días desde la realización del renograma. Finalmente se obtuvo una muestra de 54 sujetos, con edades comprendidas entre los 19 y los 80 años (media 51.8 + 29.06 años; IC 95\%: 47.9-55.7), y se capturaron 8 diagnósticos de envío (Tabla 1). Se calculó una potencia del $86 \%$ de acuerdo a la muestra final obtenida en intención a tratar y del $94 \%$ por protocolo, para un alfa de 0.05 , por método de dos proporciones basado en la media de sesgo encontrada, en contraste con los resultados de Arreola-Guerra, et al. Las medias de las mediciones y estimación por rubro se muestran en la tabla 2.

El acuerdo entre métodos resultó significativamente negativo, con un coeficiente de determinación $\left(R^{2}\right)$ de 0.6238 para CKD-EPI vs. renograma dinámico ${ }^{99 \mathrm{mT} T C-}$ DTPA $(p=0.001)$, y $R^{2}=0.6022$ para MDRD vs. renograma dinámico ${ }^{99 m T c-D T P A ~}(p=0.029)$ (Fig. 1).

El sesgo medio para la TFGe por CKD-EPI fue de $-11.39+19.47 \mathrm{ml} / \mathrm{min} / 1.73 \mathrm{~m}^{2}$ (IC 95\%:-16.59 a -6.20; $p<0.001$ ), sesgo medio para MDRD fue de $-7.38+19.18 \mathrm{ml} / \mathrm{min} / 1.73 \mathrm{~m}^{2}$ (IC 95\%: -12.49 a -2.26 ; $\mathrm{p}=0.008)$ (Tabla 3).

La precisión media para TFGe por CKD-EPI fue de $-28 \%+57 \%$ (IC 95\%: $-43 \%$ a $-12 \%$; $p<0.001$ ), precisión media para TFGe por MDRD fue de $-19 \%+$ 51\% (IC 95\%: -33\% a -6\%; p 0.008) (Tabla 3).

La exactitud $\mathrm{P}(30)$ para CKD-EPI resultó del $57 \%$, contra la exactitud $\mathrm{P}(30)$ por MDRD del $61 \%(p<0.001)$; y la exactitud $P(10)$ para CKD-EPI fue del $17 \%$, contra la exactitud $P(10)$ por MDRD del $20 \%(p<0.001)$ (Fig. 2).

La concordancia se expresa por medio del estadístico de Bland-Altman (Figs. 3 y 4).

Se identificó, además, sesgo y poca precisión en la clasificación de la ERC por grados de acuerdo a KDIGO con respecto a los métodos de estimación contra la medición por renograma dinámico, y se alcanzó una sobreestimación de hasta el $267 \%$ de los casos clasificados en estadios tempranos de ERC por CKD-EPI $(p<0.001)$ y del $200 \%$ por MDRD $(p<0.001)$, hasta el $75 \%$ de infraestimación en estadio 3a por CKD-EPI $(p<0.001)$ y del 50\% por MDRD $(p<0.001)$; destaca no haber diferencia entre métodos para la clasificación de estadio 5 (Tabla 4).

\section{Discusión}

Es evidente que la ausencia de un marcador endógeno de filtración glomerular preciso, exacto y no
Tabla 1. Características demográficas

\begin{tabular}{|c|c|}
\hline $\begin{array}{l}\text { Sexo } \\
\text { Femenino \% (n) }\end{array}$ & $67(36)$ \\
\hline $\begin{array}{l}\text { Edad } \\
\qquad \text { Media (años) }\end{array}$ & $51.8 \pm 29.06$ \\
\hline $\begin{array}{l}\text { Grupo } \% \text { (n) } \\
18 \text { a } 34 \text { años } \\
35 \text { a } 49 \text { años } \\
50 \text { a } 64 \text { años } \\
65 \text { a } 79 \text { años } \\
80 \text { años y más }\end{array}$ & $\begin{array}{c}14.8(8) \\
29.6(16) \\
35.2(19) \\
18.5(10) \\
1.8(1)\end{array}$ \\
\hline $\begin{array}{l}\text { Diagnóstico de envío \% (n) } \\
\text { Uropatía obstructiva } \\
\text { Exclusión renal* } \\
\text { ERC } \\
\text { Trasplantado renal } \\
\text { LRA } \\
\text { Candidato donador renal } \\
\text { Reflujo vesicoureteral } \\
\text { Nefrectomía } \\
\text { No especificado }\end{array}$ & $\begin{array}{c}31.5(17) \\
9.2(5) \\
7.4(4) \\
5.5(3) \\
1.8(1) \\
1.8(1) \\
1.8(1) \\
1.8(1) \\
38.8(21)\end{array}$ \\
\hline
\end{tabular}

*Exclusión funcional de uno de los riñones secundaria a patología intrarrenal (litiasis, infecciosas, degenerativas, etc.).

ERC: enfermedad renal crónica; LRA: lesión renal aguda (definición KDIGO 2012).

Tabla 2. Mediciones

\begin{tabular}{|c|c|}
\hline & Media (DE) \\
\hline Creatinina sérica (mg/dl) & $1.63 \pm 2.82$ \\
\hline TFGe CKD-EPI (ml/min/1.73 m²) & $61.62 \pm 31.72$ \\
\hline TFGe MDRD (ml/min/1.73 m²) & $57.61 \pm 30.39$ \\
\hline $\begin{array}{l}\text { TFGm renograma dinámico } \\
\text { 99mTc-DTPA }\left(\mathrm{ml} / \mathrm{min} / 1.73 \mathrm{~m}^{2}\right)\end{array}$ & $50.23 \pm 24.23$ \\
\hline
\end{tabular}

invasivo continúa siendo un factor limitante en la evaluación de la función renal, sobre todo en situaciones donde la estimación de la TFG por ecuaciones no permite su estudio de manera fidedigna, aunando las deficiencias ya documentadas para su aplicación fuera del ámbito donde fueron concebidas. Ya se han propuesto diversas proteínas de bajo peso molecular para este fin, sin embargo, no cumplen todos los criterios de una sustancia ideal para la medición del filtrado glomerular, ya que su producción no es constante, presentan aclaramiento extrarrenal y están afectadas por desórdenes inmunológicos, vitamínicos y tumorales, entre otros $^{4,5}$. Por ello, la cistatina C, la cual a priori no presenta estas limitaciones, es la proteína de bajo peso molecular que mayor interés ha despertado entre la comunidad científica y ha sido identificada como una 

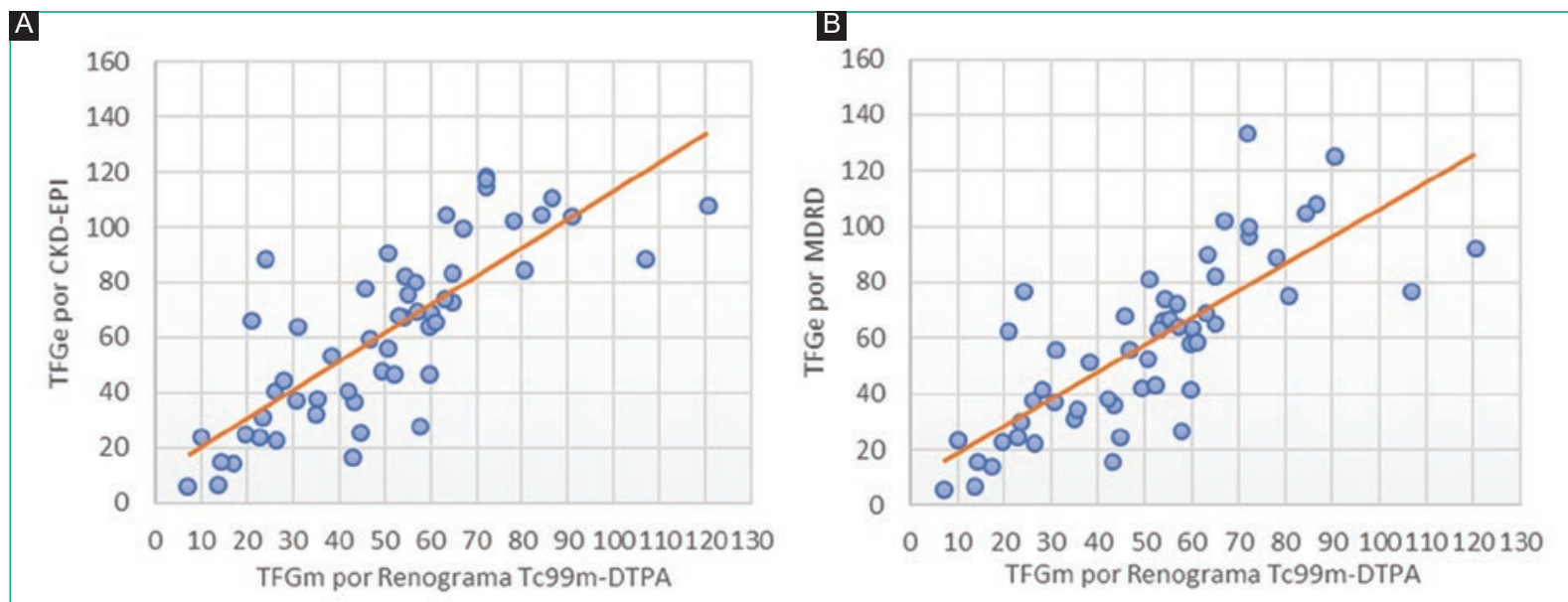

Figura 1. Correlación entre la TFGm por renograma dinámico 99mTc-DTPA y las ecuaciones de estimación: TFGe CKD-EPI $\left(r^{2}=0.6238 ; p\right.$ 0.001); TFGe MDRD ( $r^{2}=0.6022 ;$ p 0.029). CKD-EPI: Chronic Kidney Disease Epidemiology Collaboration; MDRD: Modification of Diet in Renal Disease Study, TFG: tasa de filtrado glomerular expresada en $\mathrm{ml} / \mathrm{min} / 1.73 \mathrm{~m}{ }^{2}$; TFGe: tasa de filtrado glomerular estimada; TFGm: tasa de filtrado glomerular medida.
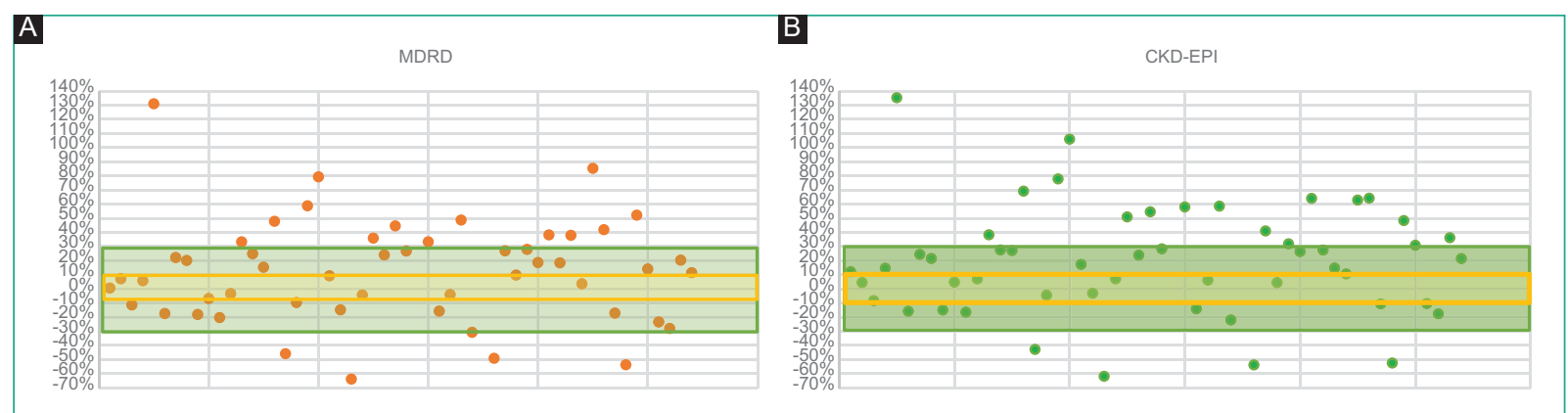

Figura 2. Exactitud $P(30)$ y $P(10)$ de CKD-EPI y MDRD. La exactitud $P(30)$ para CKD-EPI fue del $57 \%$, contra la exactitud $\mathrm{P}(30)$ por MDRD del $61 \%$ (área verde) ( $\mathrm{p}<0.001$ ); la exactitud $\mathrm{P}(10)$ para CKD-EPI fue del $17 \%$, contra exactitud $\mathrm{P}(10)$ por MDRD del 20\% (área amarilla) ( $p<0.001$ ). CKD-EPI: Chronic Kidney Disease Epidemiology Collaboration; MDRD: Modification of Diet in Renal Disease Study, P (30) y P (10): porcentaje de las mediciones a menos del 30 y $10 \%$, respectivamente, de la tasa de filtrado glomerular medida; TFGm: tasa de filtrado glomerular medida.

herramienta diagnóstica superior a la creatinina sérica en la detección de una alteración precoz de la función renal ${ }^{30}$. En 2012, el equipo de investigadores de CKDEPI desarrolló dos nuevas ecuaciones basadas en cistatina $C$ sérica y cistatina + creatinina (CKD-EPI cystatin C y CKD-EPI creatinine-cystatin C, respectivamente) mediante la misma metodología que su predecesora, con resultados que demostraron la superioridad de estas con respecto a las ecuaciones de estimación de la TFG basadas solo en la creatinina sérica ${ }^{31}$. En 2013, la KDIGO emitió la recomendación de su empleo de manera complementaria con alto nivel de evidencia ${ }^{1}$. No obstante, su uso aún no se encuentra disponible en la gran mayoría de centros hospitalarios, y continúa fuera de los cuadros básicos de atención por su alto costo y poca accesibilidad.

Por lo cual, cobra suma relevancia el desarrollo de nuevos métodos de evaluación de la función renal, más asequibles y con alta sensibilidad, sobre todo en individuos de origen hispano de los que se cuenta con tan poca evidencia, especialmente en población anciana $^{21-23,32}$.

Destaca que en México, así como alrededor del mundo, han sido escasas las publicaciones destinadas para demostrar el funcionamiento de las ecuaciones basadas en la creatinina sérica, sea por desconocimiento 
Nefro Latinoam. 2020;17

Tabla 3. Sesgo y precisión de las ecuaciones de estimación con respecto a TFG medida por renograma

\begin{tabular}{|l|c|c|c|c|}
\hline & TFGe CKD-EPI & IC 95\% & TFGe MDRD & IC 95\% \\
\hline Sesgo $\left(\mathrm{ml} / \mathrm{min} / 1.73 \mathrm{~m}^{2}\right)$ & $-11.39 \pm 19.47$ & $(-16.59 \mathrm{a}-6.20)$ & $-7.38 \pm 19.18$ & $\begin{array}{c}(-12.49 \mathrm{a}-2.26) \\
\mathbf{p = 0 . 0 0 8}\end{array}$ \\
\hline Precisión (\%) & & $\mathbf{p}<\mathbf{0 . 0 0 1}$ & & $(-33 \mathrm{a}-6)$ \\
$\mathbf{p}=\mathbf{0 . 0 0 8}$
\end{tabular}

CKD-EPI: Chronic Kidney Disease Epidemiology Collaboration; ERC: enfermedad renal crónica; MDRD: Modification of Diet in Renal Disease Study, TFG: tasa de filtrado glomerular expresada en $\mathrm{ml} / \mathrm{min} / 1.73 \mathrm{~m}^{2}$; TFGe: tasa de filtrado glomerular estimada; TFGm: tasa de filtrado glomerular medida.

Tabla 4. Correlación en la clasificación de ERC

\begin{tabular}{|c|c|c|c|c|c|c|c|}
\hline & \multirow{2}{*}{$\begin{array}{l}\text { TFGe CKD-EPI } \\
\%(n) n=54\end{array}$} & \multirow{2}{*}{$\begin{array}{l}\text { TFGe MDRD } \\
\%(n) n=54\end{array}$} & \multirow{2}{*}{$\begin{array}{l}\text { TFGm Renograma dinámico } \\
\text { 99mTc-DTPA \% (n) n = 54 }\end{array}$} & \multicolumn{2}{|c|}{ TFGe CKD-EPI } & \multicolumn{2}{|c|}{ TFGe MDRD } \\
\hline & & & & Sesgo & Precisión & Sesgo & Precisión \\
\hline KDIGO 1 & $20.3(11)$ & $16.6(9)$ & $5.5(3)$ & -8 & $-267 \%$ & -6 & $-200 \%$ \\
\hline KDIGO 2 & $33.3(18)$ & $31.4(17)$ & $25.9(14)$ & -4 & $-29 \%$ & -3 & $-21 \%$ \\
\hline KDIGO 3a & $5.5(3)$ & $11.1(6)$ & $22.2(12)$ & 9 & $75 \%$ & 6 & $50 \%$ \\
\hline KDIGO 3b & $20.3(11)$ & $20.3(11)$ & $22.2(12)$ & 1 & $8 \%$ & 1 & $8 \%$ \\
\hline KDIGO 4 & $12.9(7)$ & $14.8(8)$ & $16.6(9)$ & 2 & $22 \%$ & 1 & $11 \%$ \\
\hline KDIGO 5 & $7.4(4)$ & $5.5(3)$ & $7.4(4)$ & 0 & $0 \%$ & 1 & $25 \%$ \\
\hline
\end{tabular}

CKD-EPI: Chronic Kidney Disease Epidemiology Collaboration; KDIGO: Kidney Disease: Improving Global Outcomes; MDRD: Modification of Diet in Renal Disease Study, TFG: tasa de filtrado glomerular expresada en $\mathrm{ml} / \mathrm{min} / 1.73 \mathrm{~m}^{2}$; TFGe: tasa de filtrado glomerular estimada; TFGm: tasa de filtrado glomerular medida.

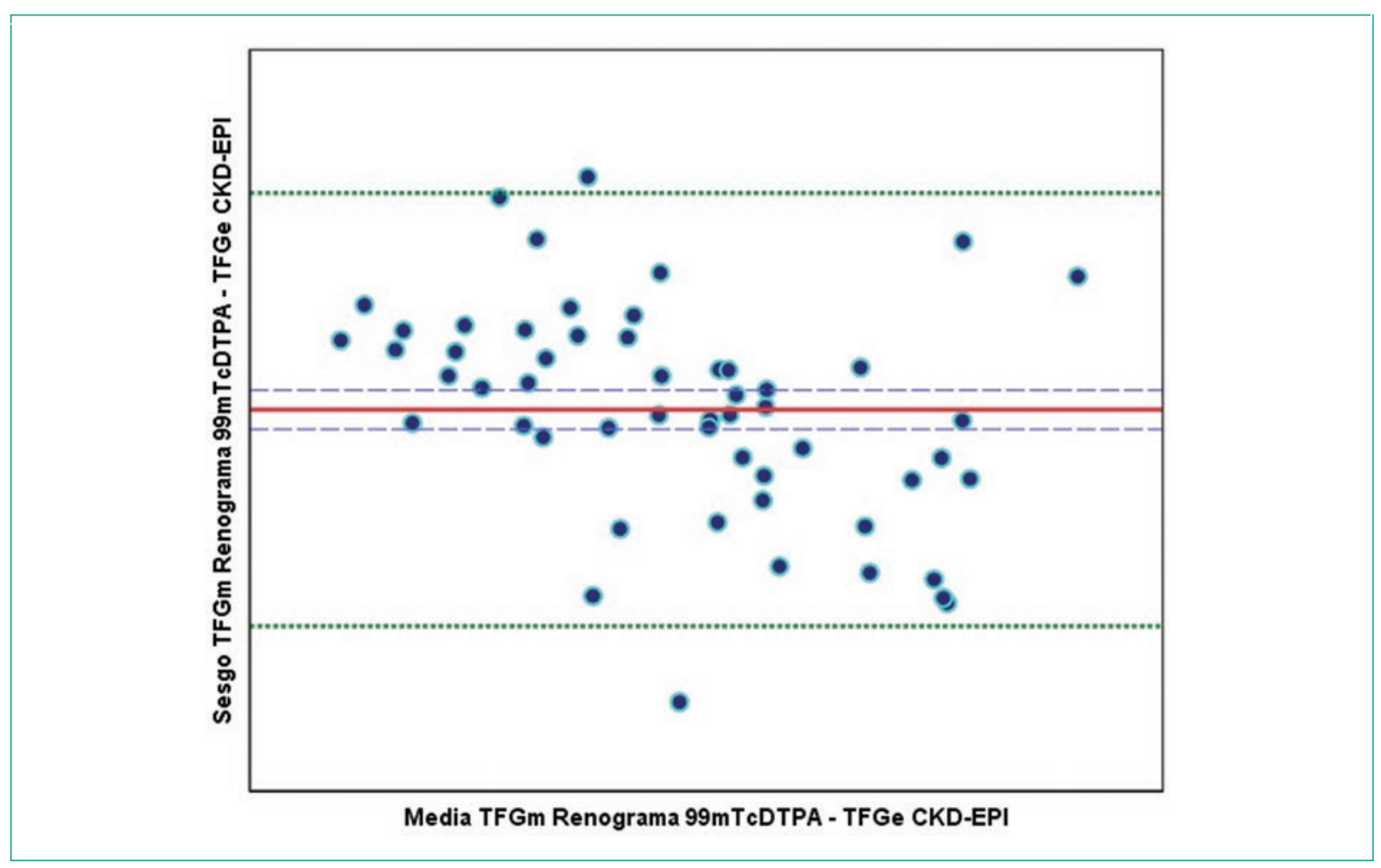

Figura 3. Gráfico de Bland-Altman: concordancia entre CKD-EPI y renograma dinámico ${ }^{99 m}$ Tc-DTPA. Línea roja: media de concordancia (media de sesgo = $-11.39 \mathrm{ml} / \mathrm{min} / 1.73 \mathrm{~m}^{2}$ ); entre líneas punteadas verdes: IC 95\% (dispersión permisible $\mathrm{a} \pm 2 \mathrm{DE}$ desde media $= \pm 38.94 \mathrm{ml} / \mathrm{min} / 1.73 \mathrm{~m}^{2}$ ); entre líneas discontinuas azules: sesgo máximo permitido (determinado en $\pm 3.5 \mathrm{ml} / \mathrm{min} / 1.73 \mathrm{~m}^{2}$ ). CKD-EPI: Chronic Kidney Disease Epidemiology Collaboration; DE: desviación estándar. 


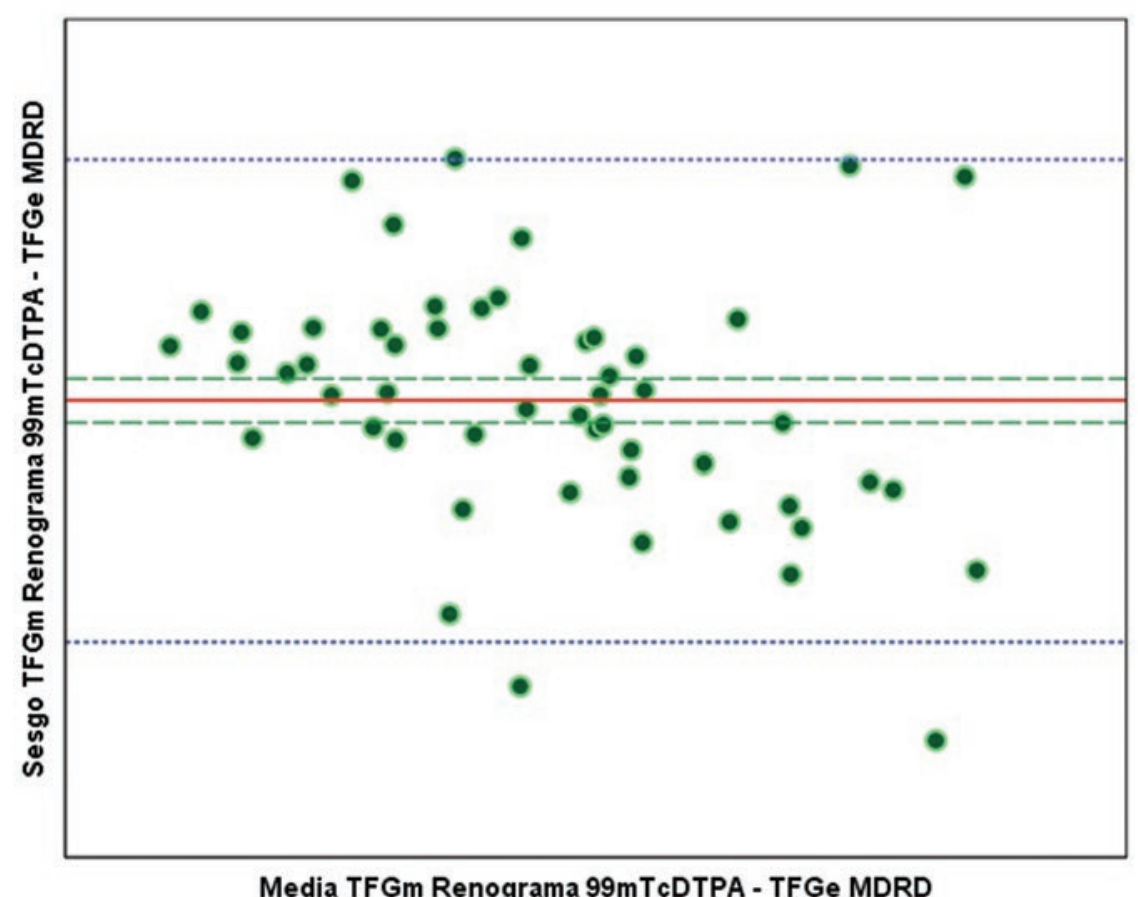

Figura 4. Gráfico de Bland-Altman: concordancia entre MDRD y renograma dinámico ${ }^{99 m}$ Tc-DTPA. Línea roja: media de concordancia (media de sesgo $=-7.38 \mathrm{ml} / \mathrm{min} / 1.73 \mathrm{~m}^{2}$ ); entre líneas punteadas azules: IC 95\% (dispersión permisible a $\pm 2 \mathrm{DE}$ desde media $= \pm 38.36 \mathrm{ml} / \mathrm{min} / 1.73 \mathrm{~m}^{2}$ ); entre líneas discontinuas verdes: sesgo máximo permitido (determinado en $\left.\pm 3.5 \mathrm{ml} / \mathrm{min} / 1.73 \mathrm{~m}^{2}\right)$. MDRD: Modification of Diet in Renal Disease Study, DE: desviación estándar.

de la prevalencia real de pacientes con ERC ante la falta de un registro nacional de enfermos renales ${ }^{33-38}$, o por el empleo generalizado e indiscriminado de dichas ecuaciones en la práctica clínica cotidiana, lo que incide directamente en la capacidad de detección y seguimiento de la enfermedad renal, sobre todo en los grupos más susceptibles.

Este primer estudio, por su carácter de piloto, si bien no refleja la generalidad de la población susceptible de estudio (etnia hispana), específicamente para aquellos sujetos portadores de ERC, mantiene correlación en sus resultados con el resto de publicaciones de similares características en distintos grupos poblacionales, que demuestran la falta de funcionalidad de las ecuaciones MDRD y CKD-EPI, con el impacto epidemiológico que evidentemente conlleva el empleo de una herramienta de evaluación clínica sesgada. Así mismo, y a diferencia del estudio realizado por Arreola-Guerra, et al., la ecuación MDRD, y no la CKD-EPI, es la que demuestra mejor funcionalidad. Esto probablemente es secundario a la población que se ha estudiado (100\% sanos para el estudio de Arreola-Guerra contra el $96 \%$ portadores de ERC en el presente estudio) y pudiera ser esperado por la selección de pacientes en los estudios pioneros (MDRD en población exclusivamente nefrópata, CKDEPI). Se han encontrado resultados similares en estudios que evalúan otras poblaciones que aúnan comorbilidades con la ERC, como cirrosis, hipertensión arterial sistémica, diabetes mellitus, entre otras, y encuentran un mejor funcionamiento para la estimación de la TFG con MDRD que con CKD-EPI ${ }^{39-41}$.

El renograma dinámico con ${ }^{99 m}$ Tc-DTPA, como método de medición de la TFG, se ha utilizado como estándar de oro en diversos estudios a nivel mundial para la validación de las ecuaciones basadas en la creatinina sérica, e incluso en la cistatina ${ }^{42,43}$, al poseer por sí solo una sensibilidad comparable con una sobreestimación de la TFG media de tan solo $3.5 \mathrm{ml} / \mathrm{min}$ con respecto al aclaramiento de inulina ${ }^{6}$. Tiene la ventaja también de ser poco invasivo, ya que solo requiere la instilación del radiofármaco en dosis bajas (185 MBq), y no es necesaria la obtención de muestras sanguíneas. Además, no se reconocen contraindicaciones absolutas, el nivel de sesgo operador-dependiente es muy bajo, los eventos adversos se asocian solo a hipersensibilidad (muy rara) al radiofármaco y los resultados 
pueden ser descritos en tiempo real. Destaca también que, en México, se emplea con regularidad para la evaluación de candidatos a donación renal. No obstante, no está exento de sesgos, de índole técnica en su mayoría (al basar la medición de la sustancia radioactiva en el aclaramiento de creatinina, el método de Gates es susceptible de demostrar las mismas deficiencias, además, se ha determinado que el tecnecio incluido en DPTA, pese a que es eliminado por vía renal de manera exclusiva, se une a proteínas plasmáticas en un 5 a $10 \%$ de su concentración total, a diferencia de la inulina, lo que infraestima en la misma medida la TFG) $)^{42,44}$, y ya han sido publicados algunos estudios que ponen en duda su precisión, sobre todo en comparación con el método de aclaramiento de ${ }^{99 \mathrm{~m} T \mathrm{~T} \text { - }}$ DTPA, entre los que destaca un estudio realizado en China que analizó a 551 pacientes sanos y con ERC (274 y 277, respectivamente), donde se demuestra que las ecuaciones por CKD-EPI y MDRD son superiores en exactitud y precisión en esta población, particularmente la sana, en comparación con el renograma dinámico, sin embargo, inferiores para los sujetos con TFG $<60 \mathrm{ml} / \mathrm{min} / 1.73 \mathrm{~m}^{2}$. Agrega, además, que la utilidad del renograma dinámico con ${ }^{99 \mathrm{~m} T c-D T P A}$ en estos grupos puede ser superior para discernir de manera confiable entre los grados $3 a$ y $3 \mathrm{~b}$ de $\mathrm{ERC}^{45}$, evidencia que se reproduce en el presente estudio.

Reconocemos que el presente estudio tiene como principal debilidad su tamaño muestral y su carácter retrospectivo. Si bien la muestra es superior en número a la media encontrada en otros estudios piloto, la bastedad del universo que plantea la problemática incide de manera importante sobre la aplicación práctica de los resultados reportados. Así mismo, la variabilidad de diagnósticos en la población estudiada y también su grupo etario, tienen un papel importante como variables bien reconocidas como modificadores en la estimación de la TFG, y pudieron haber incidido de manera significativa en los hallazgos reportados. No obstante, nuestros resultados guardan una estrecha relación con las distintas publicaciones realizadas en otros países, que reportan una falta de funcionamiento de las ecuaciones diseñadas en su mayoría sobre una población específica que no necesariamente se relaciona a otras regiones, razas, etc. Destacamos, además, que la nuestra es una de las pocas investigaciones con este respecto en el ámbito latinoamericano, y que ha facilitado el diseño de un estudio a gran escala (en curso actualmente) que debe contribuir a comprender y analizar las variables que pudieran incidir en la funcionalidad de dichas ecuaciones sobre esta población específica, idealmente multicéntrico y con validación externa con estudios que demuestren resultados similares con el empleo de un estándar de oro por aclaramiento estandarizado, que así mismo, y de acuerdo a los resultados obtenidos, permita el desarrollo de una nueva ecuación de estimación o adecuaciones a las ya existentes para su empleo de manera generalizada en población de origen hispano, con y sin enfermedad renal e independiente del diagnóstico etiológico en la mayor medida posible, siendo este el propósito final de la investigación.

\section{Conclusiones}

Ambas ecuaciones de estimación de la TFG basadas en la creatinina sérica (CKD-EPI y MDRD), sobreestiman de manera significativa la TFG de los pacientes mexicanos estudiados, independientemente del sexo, la edad y el diagnóstico, con y sin ERC.

La ecuación MDRD tiene mejor funcionalidad que la CKD-EPI, sobre todo en aquellos que cursan con ERC, excepto en grado 5 .

Como estudio preliminar, destacamos que es necesaria la realización de estudios de mayor escala, potencia y con validación externa, para confirmar nuestros resultados, así como para identificar aquellas variables que incidan directamente en la funcionalidad de las ecuaciones de estimación de la TFG en población hispana.

\section{Conflicto de intereses}

Los autores declaran no tener ningún conflicto de intereses.

\section{Responsabilidades éticas}

Protección de personas y animales. Los autores declaran que para esta investigación no se han realizado experimentos en seres humanos ni en animales.

Confidencialidad de los datos. Los autores declaran que han seguido los protocolos de su centro de trabajo sobre la publicación de datos de pacientes.

Derecho a la privacidad y consentimiento informado. Los autores han obtenido el consentimiento informado de los pacientes y/o sujetos referidos en el artículo. Este documento obra en poder del autor de correspondencia.

\section{Bibliografia}

1. Kidney Disease: Improving Global Outcomes (KDIGO) CKD Work Group. KDIGO 2012 Clinical Practice Guideline for the Evaluation and Management of Chronic Kidney Disease. Kidney Inter. 2013;3:1-150. 
2. Levey AS, Stevens LA, Schmid $\mathrm{CH}$, Zhang YL, Castro AF $3^{\text {rd }}$, Feldman $\mathrm{HI}$, et al. A New Equation to Estimate Glomerular Filtration Rate. Ann Intern Med. 2009;150(9):604-12.

3. Johonson RJ, Feehally J, Jurgen F. Comprehensive Clinical Nephrology. $3^{\text {rd }}$ ed. Philadelphia: Mosby; 2007.

4. Pérez-Loredo J, Lavorato C, Negri AL, Der M, Lercari J, Casaliba A. La Estimación del filtrado glomerular. Nephrol Dial Transplant. 2010; 30(3):118-29.

5. Donadio C, Lucchesi A, Ardini M, Giordani R. Cystatin C. B2-microglobulin, and retinol binding proteins as indicators of glomerular filtration rate: comparison with plasma creatinine. J Pharm Biom Anal. 2001;24:835-42.

6. Rehling M, Møller ML, Thamdrup B, Lund JO, Trap-Jensen J. Simultaneous measurement of renal clearance and plasma clearance of 99mTc-labelled DTPA, 51Cr-labelled EDTA and inulin in man. Clin Sci. 1998; 66:613-9.

7. Levey AS, Berg RL, Gassmann JJ, Hall PM, Walker WG. Creatinine filtration, secretion and excretion during progressive renal disease. Kidney Int. 1989;36(27):S73-80.

8. Levey AS. Measurement of renal function in chronic renal disease. Kidney Int. 1990;38:167-84

9. Secretaría de Salud. Guía de Práctica Clínica Prevención, Diagnóstico y Tratamiento de la Enfermedad Renal Crónica Temprana. CENETEC México; 2009

10. Fraser SDS, Aitken G, Taal MW, Mindell JS, Moon G, Day J, et al. Exploration of Chronic Kidney Disease Prevalence Estimates Using New Measures of Kidney Function in the Health Survey for England. PLoS One. 2015;10(2):e0118676.

11. Cockcroft DW, Gault MH. Prediction of creatinine clearance from serum creatinine. Nephro. 1976;16:31-41.

12. Dineshan KM, Shenoy A, Cardoza F. Accuracy of Various Creatinine Based Prediction Equations For Estimating Glomerular Filtration Rate in Prospective Donors in Comparison to DTPA Renogram. IOSR-JDMS. 2017; 16:01-06.

13. Levey AS, Bosch JP, Lewis JB, Greene T, Rogers N, Roth D. A more accurate method to estimate glomerular filtration rate from serum creatinine: a new prediction equation. Modification of Diet in Renal Disease Study Group. Ann Intern Med. 1999;130:461-70.

14. Zuo L, Ma YC, Zhou YH, Wang M, Xu GB, Wang HY. Application of GFR-estimating equations in Chinese patients with chronic kidney disease. Am J Kidney Dis. 2005;45(3):463-72.

15. Rule AD, Larson TS, Bergstralh EJ, Slezak JM, Jacobsen SJ, Cosio FG Using serum creatinine to estimate glomerular filtration rate: Accuracy in good health and in chronic kidney disease. Ann Intern Med. 2004; 141(12):929-37.

16. Shafi T, Marsushita K, Selvin E, Sang Y, Aston BC, Inker LA, et al. Comparing the association of GFR estimated by CKD EPI and MDRD equations and mortality: the third national health and nutrition examination survey (NHANES III). BMC Nephrol. 2012;13:42.

17. Alaini A, Malhotra D, Rondon-Berrios H, Argyropoulos CP, Khitan ZJ, Raj DSC, et al. Establishing the presence or absence of chronic Kidney disease: Uses and limitations of formulas estimating the glomerular filtration rate. World J Methodol. 2017:7(3):73-92

18. Stevens LA, Schmind $\mathrm{CH}$, Zhang YL, Coresh J, Manzi J, Landis R, et al Development and validation of GFR-estimating equations using diabetes, transplant and weight. Nephrol Dial Transplant. 2010;25:449-57.

19. Stevens LA, Claybon MA, Shmid CH, Chen J, Horio M, Imai E, et al. Evaluation of the Chronic Kidney Disease Epidemiology Collaboration equation for estimating the glomerular filtration rate in multiple ethnicities. Kidney Int. 2011;79:555-62.

20. Martínez-Martínez MU, Borjas-García JA, Llamazares-Azuara L, Torres-Anguiano JR, Hernández-Nieto CA, Abud-Mendoza C. Evaluación de la función renal en pacientes con lupus eritematoso sistémico (LES) por ecuación basada en creatinina sérica, correlación con iodotalamato y cistatina $\mathrm{C}$. Inutilidad de la depuración de creatinina (DCr). Reumatol Clín. 2011;7(1):81

21. Gámez-Jiménez AM, Montell-Hernández OA, Ruano-Quintero $V$ de León A, Hay de la Puente-Zoto M. Enfermedad renal crónica en el adulto mayor. Rev Med Electrón. 2013:35(4):306-18.

22. Fan L, Levey AS, Gudnason V, Eiriksdottir G, Andresdottir MB, Gudmundsdottir $\mathrm{H}$, et al. Comparing GFR estimating equations using cystatin $\mathrm{C}$ and creatinine in elderly individuals. J Am Soc Nephrol. 2014:1-8.

23. Mora-Gutiérrez JM, Slon-Roblero MF, Castaño-Bilbao I, Izquierdo-Bautista D, Arteaga Coloma J, Martínez-Velilla N. Enfermedad renal crónica en el paciente anciano. Rev Esp Geriatr Gerontol. 2017;52:152-8.

24. Schaeffner ES, Ebert N, Delanaye P, Frei U, Gaedeke J, Jakob O, et al. Two novel equations to estimate kidney function in persons aged 70 years or older. Ann Intern Med. 2012;157:471-81.
25. Horio M, Imai E, Yasuda Y, Watanabe T, Matsuo S. Modification of the CKD Epidemiology Collaboration (CKD-EPI) Equation for Japanese: Accuracy and Use for Population Estimates. Am J Kidney Dis. 2010;56:32-8.

26. Liu X, Yanni Wang, Cheng Wang, Chenggang Shi, Cailian Cheng, et al. A New Equation to Estimate Glomerular Filtration Rate in Chinese Elderly Population. PLoS One. 2013;8:e79675.

27. Rule AD, Larson TS, Bergstralh EJ, Slezak JM, Jacobsen SJ, Cosio FG. Using serum creatinine to estimate glomerular filtration rate: Accuracy in good health and in chronic kidney disease. Ann Intern Med. 2004; 141(12):929-37.

28. Rigalleau V, Lasseur C, Perlemoine C, Barthe N, Chauveau P, Combe C, Gin $\mathrm{H}$. The Mayo clinic quadratic equation improves the prediction of glomerular filtration rate in diabetic subjects. Nephrol Dial Transplant. 2007:22(3):813-18

29. Arreola-Guerra JM, Rincón-Pedrero R, Cruz-Rivera C, Belmont-Pérez T, Correa-Rotter R, Niño-Cruz JA. Funcionamiento de las fórmulas MDRD-IDMS y CKD-EPI, en individuos mexicanos con función renal normal. Nefrología. 2014;34(5):591-8.

30. Fernández-García M, Coll E, Ventura-Pedret S, Bermudo-Guitarte C, Cárdenas-Fernández MC, Cortés-Rius $\mathrm{M}$, et al. Cistatina $\mathrm{C}$ en la evaluación de la función renal. Rev Lab Clin. 2011;4(1):50-62.

31. Inker LA, Schmid CH, Tighiouart H, Eckfeldt JH, Feldman HI, Greene T, et al. Estimating Glomerular Filtration Rate from Serum Creatinine and Cystatin C. N Engl J Med. 2012;367:20-9.

32. Epstein M. Aging and the kidney. J Am Soc Nephrol. 1996;7:1106-22.

33. Espinoza-Cuevas MA. Enfermedad Renal. Gac Med Mex. 2016;152(1):90-6.

34. Lastiri-Quirós HS. Enfermedad renal crónica en México: una política nacional todavía pendiente. En: Tamayo-y-Orozco JA, Lastiri-Quirós HS. La enfermedad renal crónica en México, hacia una política nacional para enfrentarla. 1. ${ }^{a}$ ed. México: Academia Nacional de Medicina de México (ANMM), Intersistemas; 2016. p. 1-18.

35. Obrador GT, García-García G, Villa AR, Rubilar X, Olvera N, Ferreira E, et al. Prevalence of chronic kidney Disease in the Kidney Early Evaluation Program (KEEP) México and comparison with KEEP US. Kidney Int Suppl. 2010;77(suppl 116):S2-8.

36. García-García G, Gutiérrez-Padilla AJ, Chávez-Iñiguez J, Pérez-Gómez HR, Mendoza-García M, González-De la Peña M, Tonelli M. Identifying Undetected Cases of Chronic Kidney Disease in Mexico. Targeting High-risk Populations. Arch Med Res. 2013;44:623-7.

37. Cueto-Manzano AM, Cort L, Mart R, Rojas-Campos E, Benjamin G. Prevalence of Chronic Kidney Disease in an Adult Population. Arch Med Res. 2014;45(6):507-13.

38. Instituto Nacional de Salud Pública. Encuesta Nacional de Salud y Nutrición 2012. [Internet]. México. [consultada el 21 de junio de 2018]. Disponible en http://ensanut.insp.mx/doctos/FactSheet_ResultadosNacionales14Nov.pdf

39. Chen YW, Chen HH, Wang TE, Chang CW, Chang CW, Wu CJ. Difference between CKD-EPI and MDRD equations in calculating glomerula filtration rate in patients with cirrhosis. World J Gastroenterol. 2011; 17(40):4532-8

40. Gómez Marcos MA, Rodríguez Sánchez E, Recio Rodríguez JI, Martín Cantera M, Ramos Blanes R, García Ortiz L. Diferencias de la ecuación CKD-EPI con la de MDRD para la estimación del filtrado glomerular en pacientes hipertensos. Nefrología. 2010;30(4):458-62.

41. Schwandt A, Denkinger M, Fasching P, Pfeifer M, Wagner C, Weiland J, Zeyfang A, Holl RW. Comparison of MDRD, CKD-EPI, and Cockcroft-Gault equation in relation to measured glomerular filtration rate among a large cohort with diabetes. J Diabetes Complications. 2017;31(9):1376-83.

42. Trimarchi $\mathrm{H}$, Muryan A, Martino D, Toscano A, Iriarte R, Campolo-Girard V, et al. Creatinine- vs. cystatin C-based equations compared with $99 \mathrm{mTC}$ DTPA scintigraphy to assess glomerular filtration rate in chronic kidney disease. J Nephrol. 2012;25(06):1003-15.

43. White C, Akbari A, Hussain N, Dinh L, Filler G, Lepage N, Knoll GA. Chronic kidney disease stage in renal transplantation-classification using cystatin $\mathrm{C}$ and creatinine-based equations. Nephrol Dial Transplant. 2007; 22:3013-20.

44. Gates GF. Split renal function testing using Tc-99m DTPA. A rapid technique for determining differential glomerular filtration. Clin Nucl Med. 1983;8:400-7.

45. Huang Q, Chen Y, Zhang M, Wang S, Zhang W, Cal G, Chen X, Sun X. Comparative evaluation of technetium 99 mdiethylenetriaminepentaacetic acid renal dynamic imaging versus the Modification of Diet in Renal Disease equation and the Chronic Kidney Disease Epidemiology Collaboration equation for the estimation of GFR. Int Urol Nephrol. 2018;50:733-3. 